\title{
COMPARATIVE ANALISIS OF THE FUNCTIONAL STATUS OF THE SPINE IN STUDENTS MEN AND WOMEN
}

\author{
P. Angelova ${ }^{1}$, P. Petkov ${ }^{2 *}$ \\ ${ }^{1}$ Rehabilitation Centre "Kinezi-3" \\ ${ }^{2}$ Department of Physical Education and Sport, Trakia University, Stara Zagora, Bulgaria
}

\begin{abstract}
Whether an initial curved spine will move to a higher degree and bad posture will cause health problems so far can not be safely established by certain method. It is need early diagnosis, prevention, postures control of these cases. The aim of the study was to compare the data on the state of the back muscles between men and women and to understand how existing deviations from the normal posture. Somatoskopics method for diagnosing spinal deformities served us to objectify the results of this screening. Recognize incomplete to evaluate the degree of spinal deformities, but emphasize that the use of skoliosis measuring devices, radiographic and Fluorographic photosomatoskopists laborious methods, and some of them are only applicable to heavy spurs and cases hump. Other methods are also expensive. The study was conducted with 50 students and 262 students of Trakia University - Stara Zagora at the beginning of 2013/14 school year. The determination of the functional condition of the spine is made up of several test mobility in the frontal and sagital plane. The results shown in men and women suggest that women more often measured deviation from normal . The main stabilizing muscles have reduced tone and function especially in women and men reported more limited movements and reduced mobility of the spine. The sports university with the greatest prophylactic importance in spinal deformities are basketball, volleyball and swimming, we recommend practicing. For many of the students with spinal deformities find is primary. Those who have already been diagnosed with such pathology are performed control and continuous corrective actions.
\end{abstract}

Key words: curved spine, students, poor posture, muscle imbalance.

\section{INTRODUCTION}

Approximately $2 \%$ to $3 \%$ of Americans age 16 are diagnosed with scoliosis. Less than $0.1 \%$ have a spinal curvature of more than 40 degrees. More girls than boys are affected [6]. Statistical publications show that not every spinal curvature and incorrect posture progresses in a more severe form. Whether an initial curved spine will move to a higher degree and bad posture will cause health problems so far can not be safely established by certain method. It is need early diagnosis, prevention, posture control of these cases. Causes of scoliosis is unknown which hinders prevention, but early detection can prevent the consequences of the possible progression. In Bulgaria after the changes in health diagnostic

\footnotetext{
"Correspondence to: Plamen Petkov, Trakia University Department of Physical Education and Sport, Students Campus, 6000 Stara Zagora, Bulgaria, Tel.: +359887881260, Email:jo_team@abv.bg
}

events of spinal deformities decreased to the extent of their absence. According to the Institute of physical education and school hygiene BAS performed by screening for the students set a high incidence. $30 \%$ of surveyed children were registered with spinal deformities (Koleva 2002). Due to the difference in the criteria and classification of spinal deformities and included them poor posture difficult to compare data. Another makes us an impression in a survey of the available literature is almost missing information on the frequency of the problem among students. S. Chernogorova points out that reviewed during 1980-1984 year 1,500 students from Sofia, Plovdiv and Varna $53.80 \%$ of the students and $46.20 \%$ of the students have spinal deformities with scoliosis are respectively $21.15 \%$ and $28,80 \%$. Found $8.09 \%$ with kyphosis. This leads us to conclude that the problem of diagnosis of youths 18-24 deserves attention. Many authors expose the view that the completion of the 
bone structure of the vertebrae and intervertebral spaces settled in age from 18-19 to 20-24 years. The increase of the muscle fibers of the thigh continues to 20-24. These anatomical and physiological characteristics make us think about the importance of the diagnosis of spinal deformities in high schools and their correction with adequate sports students. Proper diagnostic coverage of all features, characteristics and interconnections are crucial to detect deformities posture and spinal deformities, their treatment and prognosis [1]. Disturbed muscle balance in poor posture, poor motor pattern of standing and seated and wearing weights can lead to spinal curvature as it is decisive for their formation. Electromyographic studies and tonometrics dorsal muscles in different body positions confirm this. Dispensary for students with spinal curvature is almost a gap in the health system.

The aim of the study was to compare the data on the state of the back muscles between men and women and to understand how existing deviations from the normal posture.

To realize this purpose it is necessary to perform the following tasks:

1 . To carry out testing.

2 . To process and analyze the results.

The subject is the functional condition of the spine in students.

Object of study students and students of Thracian University Stara Zagora.

\section{METHODOLOGY}

The study was conducted with 50 students and 262 students of Trakia University - Stara Zagora at the beginning of the academic 2013/14

Attached are the following methods:

1. Theoretical analysis.

2. Interview.

3. Tetrahedron of V. H. Moshkov [4].

To measure the lateral distortion is used quadrangle of V.N. Moshkov.

4. Test for the mobility of the spine [3].

The determination of the functional condition of the spine in the various departments made up of several test mobility in the frontal and sagital plane.

5. Method of Ott [3].

Through it finds limited flexion of the thoracic segment.

6. Method of Schober [3].
This method was used to measure the amplitude of the unfolding of the lumbar segment where flexion of the spine

7. Analysis of the relative shares.

\section{RESULTS AND ANALYSIS}

The average age of respondents was 20.6 years.

The determination of the functional condition of the spine is made up of several test mobility in the frontal and sagittal plane.

For flexion in the cervical segment establish deficits in $33.46 \%$ of women and $17.65 \%$ for men. These results can be looked at (Figure 2) and (Figure 1). This suggests a strong flexor muscles of the neck in men. And we should point out the influence of immobilization posture in most of the time students. Combined with gravitational forces experienced by the body when standing, especially while sitting lead to excessively tilted forward position of the head. The test tilt forward illustrated in (Figure 2) and (Figure 1) there is a deficit of overall mobility in $27.38 \%$ of women and $33.33 \%$ for men. This deficit is not so great, but in terms of Kinesiology can conclude that it is due to shortened and rigid muscles, not only back, but the back of the thigh. This is expressed in a greater extent in men as they exercise more sports related to the load of the lower extremities. In the measurement of the waist segment in flexion of the spine by means of the mode of Schober found that $69.58 \%$ of the examined women and $66.67 \%$ of men are outside the normal range of the unfolding and this is reflected on (Figure 2) and (Figure 1). Studies of the prevalence of more pronounced lumbar lordosis suggests that it is more common in women [6]. This mobility may be a signal of a high number of young people with increased lumbar lordosis or hypotonic muscles, which can result in expression of Discogenic pathology such as spondylolisthesis, facet syndrome, compression of $\mathrm{n}$. ishiadicus, etc. In the establishment of weak musculature (in particular dorsal) attention should be sharpened, because it is a sign of predisposition to curvature of the spine [5]. In such cases it is necessary to strengthen the measures for increasing physical activity to revitalize weak back muscles, to restore normal mobility of the spine and be prevented prescoliosis and poor posture. By ways of Ott establish limited flexion of the thoracic segment. The mobility of the spine in the thoracic department is limited to $79.85 \%$ of women and $74.51 \%$ of men, as we see in (Figure 2) and (Figure 1). The demonstration 
in the case of poor functional status of muscles in the chest department is probably a result of reduced physical activity, leading to shortened and rigid back muscles. In weak back muscles (romboids and trapezuis) those in front of the body become too powerful and shortened dorsal kyphosis increases. In women, these process is demonstratively expressed. Diagnostic screening of 22,145 adolescents from 7-18 years held in Veliko Tarnovo, in which there was an increase of frequency with increasing age has served us for information regarding kyphosis. [2] We note the high percentage of reduced mobility in men which was expected to result in our study, as in the study of the problem of the presence of kyphosis men to strike. In lateral neck left and right to establish abnormal mobility in $99.62 \%$ of women and men 100\%, reflected on (Figure 2) and (Figure 1). Indicative of the condition of the cervical muscles - they contraction and spastic shortened and tense. This suggests there is an imbalance of the cervical muscles. More worrying about the state of the cervical spine is that $74 \%$ of women and $84.31 \%$ of the men was no difference in mobility between the left and right sides. Measurements of the side slopes of the body to establish a distinction between the left and right with a high proportion of respondents for women $-87.84 \%$ and for men $-82.35 \%$, which is indicated on the (Figure 2) and (Figure 1). Stronger and trained, men, and possibly a shortened muscles of the body as the wide dorsal, lumbar muscle square are the reason for the limited side slope. This difference is assumed due to the imbalance of the back muscles, presence of vicious movement patterns and postural stress, lack of fitness level in women of these important for normal posture muscles and can be a sign of spinal curvature. We used the quadrangle of V.N. Moshkov [4], which accounts for a curtailment of the extensor group back muscles and the asymmetry of the blades on top of scoliosis. It is affordable and easy method for measuring the lateral curvature of the spine. The results obtained in the test "rectangle of the Moshkov" which is formed by measuring the distance between $\mathrm{C} 7$ and lower medial angles of the blades and L5 indicate that there are two by two equal. Of (Figure 2) and (Figure 1) is visible the above parties difference $77.19 \%$ of women and $80.39 \%$ of men. For undersides differential is established at $77.95 \%$ of the women and $64.71 \%$ in men. As we have seen in lateral flexion of the neck and the trend is for men shortened muscles around the neck and shoulder blades. In women, cervical muscles and the muscles around the shoulder blades and their back muscles are weaker anatomically and if not trained to become even weaker tone. The result is likely to reach the spinal curvature. We made measurements for scoliosis with functional changes of the muscles in the presence of torsion changes brings diagnostic accuracy [5].
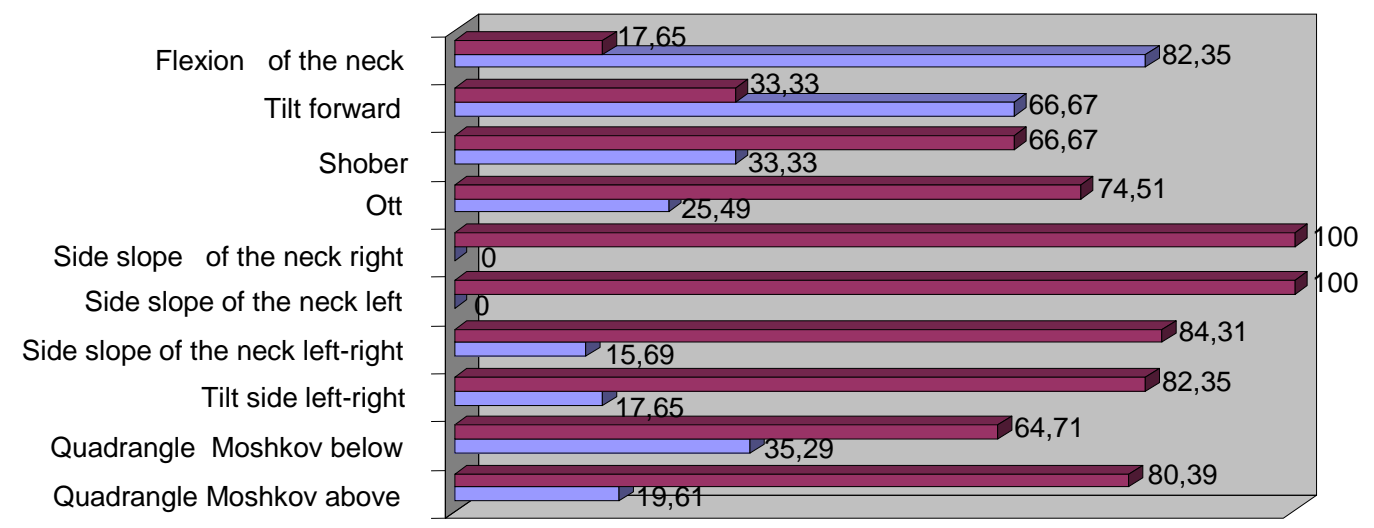

$\square$ Outside the norm

$\ominus$ Norm

Figure 1. Results of tests for men 


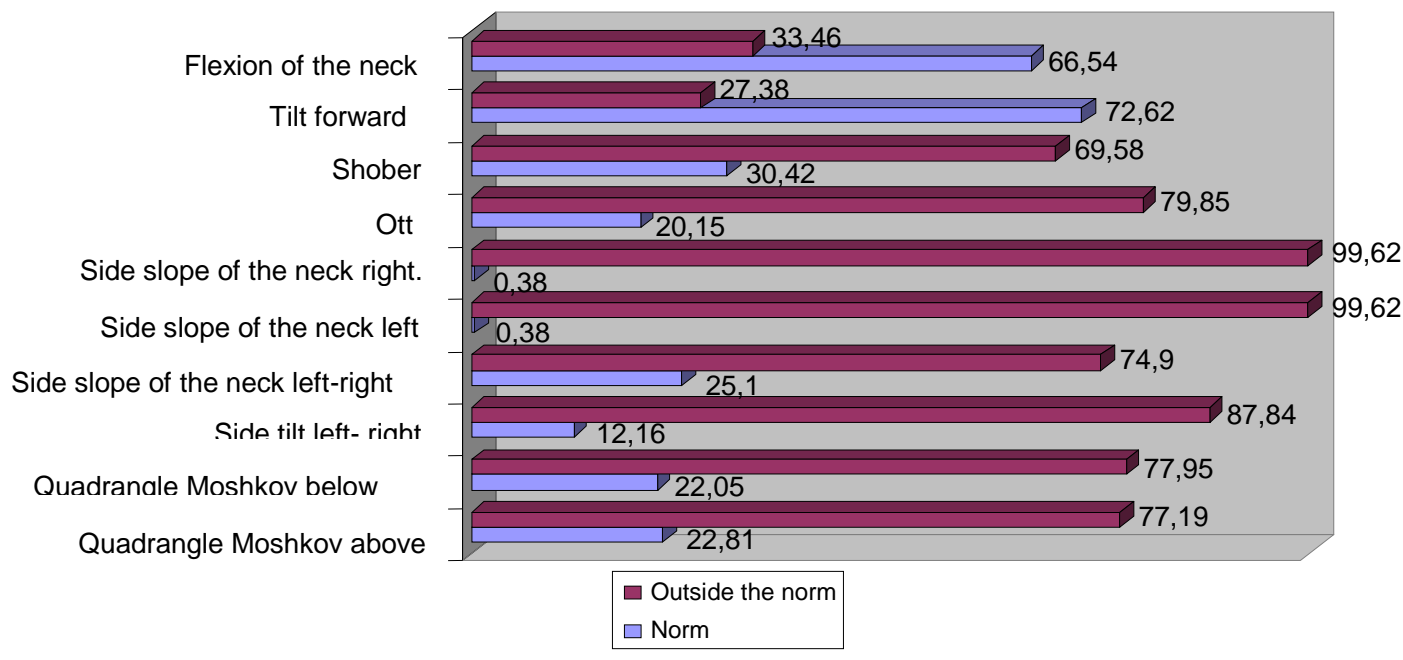

Figure 2. Results of tests for women

Somatoskopics methods used for diagnosing spinal deformities served us to objectify the results of this screening recognized as incomplete to evaluate the degree of spinal deformities, but emphasize that the use of scoliosis measuring devices, radiographic and Fluorographic, photosomatoskopics are laborintensive methods, and some of them are applicable only for severe adverse deviations and cases hump. Other methods are also expensive

\section{CONCLUSION}

In conclusion can be drawn the following conclusions and recommendations:

1. The results shown in men and women suggest that women more often measured deviation from the norm.

2. The main stabilizing muscles have reduced tone and function especially in women and men reported more limited movements and reduced mobility of the spine.

3. From sports at the university with the greatest prophylactic importance in spinal deformities are basketball, volleyball and swimming [5], we recommend practicing.

4. For many students with spinal deformities find is primary. Those who have already been diagnosed with such pathology are performed control and continuous corrective actions.

\section{REFERENCES}

1. Barakova P. Comparative analysis of the frequency distribution of spinal deformities and their course of development in modern polyclinic network. Scientific papers of the University of Ruse, t. 47, series 5.4-47, 2008.

2. Dushkov, V., N. Bakev K. Velcheva. Spinal curvature - statistics and reality. Scientific University of Rousse, Volume 51, series 8.1-20, 2012.

3. Karaneshev, D., D. Miltcheva. Diagnostics and research in therapeutic exercise. ETSNPFKS- VIF Georgi Dimitrov, S., 1984.

4. Sokolov, B., D. M. Elders. Rectifying gymnastics. Medicine and Sports, S., 1991.

5. Chernogorova, S., L. Razsolkova, D. Tsakova. Prevention of spinal curvature. Medicine and Sports, S., 1985.

6. http://www.webmd.com/osteoarthritis/guid e/arthritis-scoliosis Differences on spinal curvature in standing position by gender, age and weight status using a noninvasive method.Lang-Tapia M1, España-Romero V, Anelo J, Castillo MJ 